\title{
Smart control of gas distribution networks reliability
}

\author{
Maria Zemenkova ${ }^{1, *}$,Vitali Petryakov ${ }^{1}$, and Anton Shipovalov ${ }^{2}$ \\ ${ }^{1}$ Tyumen Industrial University, Volodarskogo str., 38, Tyumen, 625000, Russia \\ ${ }^{2}$ JSC Gazprom transgazYugorsk, Mira St., 15, Yugorsk,628260,Russia
}

\begin{abstract}
In IUT research is conducted on factors that determine the reliability and safety in the transport and storage of hydrocarbons. As the volume of gas supply increases and the system of technical regulation undergoes modernization, analysis of modern safety, reliability and energy efficiency requirements for gas networks has been conducted. Problems of ensuring reliability are especially relevant in the area of operation of gas networks and require a careful study of the matter. Current gas networks have a high operating time and heavy workload due to increased gas consumption, which leads to frequent disruptions in the supply of gas to consumers. A mathematical package for creating a multi-level facility management system of gas networks has been designed. A system approach regulated by the requirements of regulatory documents is based on the functions of assessment and planning of safety and reliability parameters of networks, optimization of the technological scheme when planning maintenance.
\end{abstract}

\section{Introduction}

As a result of modernization of the technical regulation system a set of rules and regulations is in action governing particular design and operation features of gas networks. So, the main features of the current set of rules SP 62.13330.2011* "Gas distribution systems"[1-6] are:

- priority of requirements aimed at ensuring safe and reliable operation of gas distribution networks, gas consumption and LPG facilities;

- ensuring safety requirements established by technical regulations and regulatory legal documents of federal executive bodies;

- protection of lawful rights and interests of consumers of construction products by regulating performance characteristics of gas distribution networks, gas consumption and LPG facilities;

- empowering effective application of modern technologies, new materials, especially polymer, and equipment for construction of new and rehabilitation of worn-out gas distribution networks, gas consumption and LPG facilities;

- ensuring energy saving and improving energy efficiency of buildings and structures;

\footnotetext{
*Corresponding author: muzemenkova@mail.ru
} 
The reliability of the gas supply system is the ability to continuously supply customers with the required amount of gas of the required quality at maximum safety in terms of a threat to people, infrastructure and the environment. Gas networks are quite complex and hazardous technical objects [2], and require a detailed study in terms of reliability and safety. A standard system of gas supply consists of a gas source, gas distribution network and internal gas equipment.

A modern gas network facility management system regulated by the requirements of regulatory documents is based on the functions of assessment and planning of safety and reliability parameters. Problems of ensuring reliability are especially relevant in the area of operation of gas networks and require a careful study of the matter. Current gas networks have a high operating time and heavy workload due to increased gas consumption, which leads to frequent disruptions in the supply and increased accidents.

In IUT research is conducted on the development of the reliability and safety monitoring system of gas networks. As part of the multivariate analysis tasks the following factors are taken into account: location of systems, conditions of line passage, human factor, technical condition of pipelines and equipment in dynamics considering the forecast, operating modes, results of diagnostics, conditioning and other structural and technological features. According to SP requirements, gas distribution networks of communities with a population of over 100 thousand people should be equipped with automated remote process control systems of gas distribution and gas consumption custody transfer (APCS GD).



Fig.1.Search and rescue operations at the site of home collapse [2].

Regulatory documentation [1] for designing gas pipelines instructs to implement strength calculations to determine: the thickness of the walls of pipes and fittings; longitudinal stresses the values of which shall not exceed the permissible.

In IUT researchers have been developing a modern algorithmic and mathematical complex for the design decision support system for managing the reliability of gas networks.

\section{Materials and Methods}

The authors analyzed the problems of assessing the reliability of gas distribution networks in modern conditions[1-14]. Analysis of existing GDN operation systems shows that there is a range of issues impeding the qualitative monitoring of reliability and safety.Theseproblemsmayinclude:legalaspectsandboundariesofresponsibilitiesofoperatingco mpanies; architectural features of gas networks, difficult to assess and optimize; technical features of high, medium and low pressure networks; lack of modern assessment tools and measuring devices adapted to on-line monitoring systems, as well as lack of expert systems 
that can be integrated in complexes of satellite geo-monitoring and domestic control systems with the use of mobile expert notification systems for the end user.

The complexity and the need for a system approach to assessing the reliability of gas networks is worth noting. Qualitative selection and application of mathematical models allow identifying the problem areas at an early stage.

A gas distribution network is a system of pipelines and equipment intended for transport and distribution of gas within the city or other community. Within the city pipelines are classified as main gas pipelines (MG) and distribution pipelines. Via MG gas is supplied from one area to another, and via the distribution pipelines - directly to gas consumers.

For example, gas distribution systems can be divided into groups of basic elements and stations providing direct transportation and distribution of gas flows:

1. high, medium and low pressure gas pipelines;

2. shut-off fitting units: valves and chokes with compensators which in the underground laying of gas pipelines are installed in wells;

3. hydraulic valves and condensate collectors installed on pipelines in the ground;

4. gas control station (GCS) and gas control points (GCP).

A scheme and classification algorithm of the process equipment of the gas network can be presented as a scheme in Figure 2. In IUT researchers have developed a shell concept, algorithms and mathematical models to analyze the reliability of gas distribution networks and improve the efficiency of reliability control.

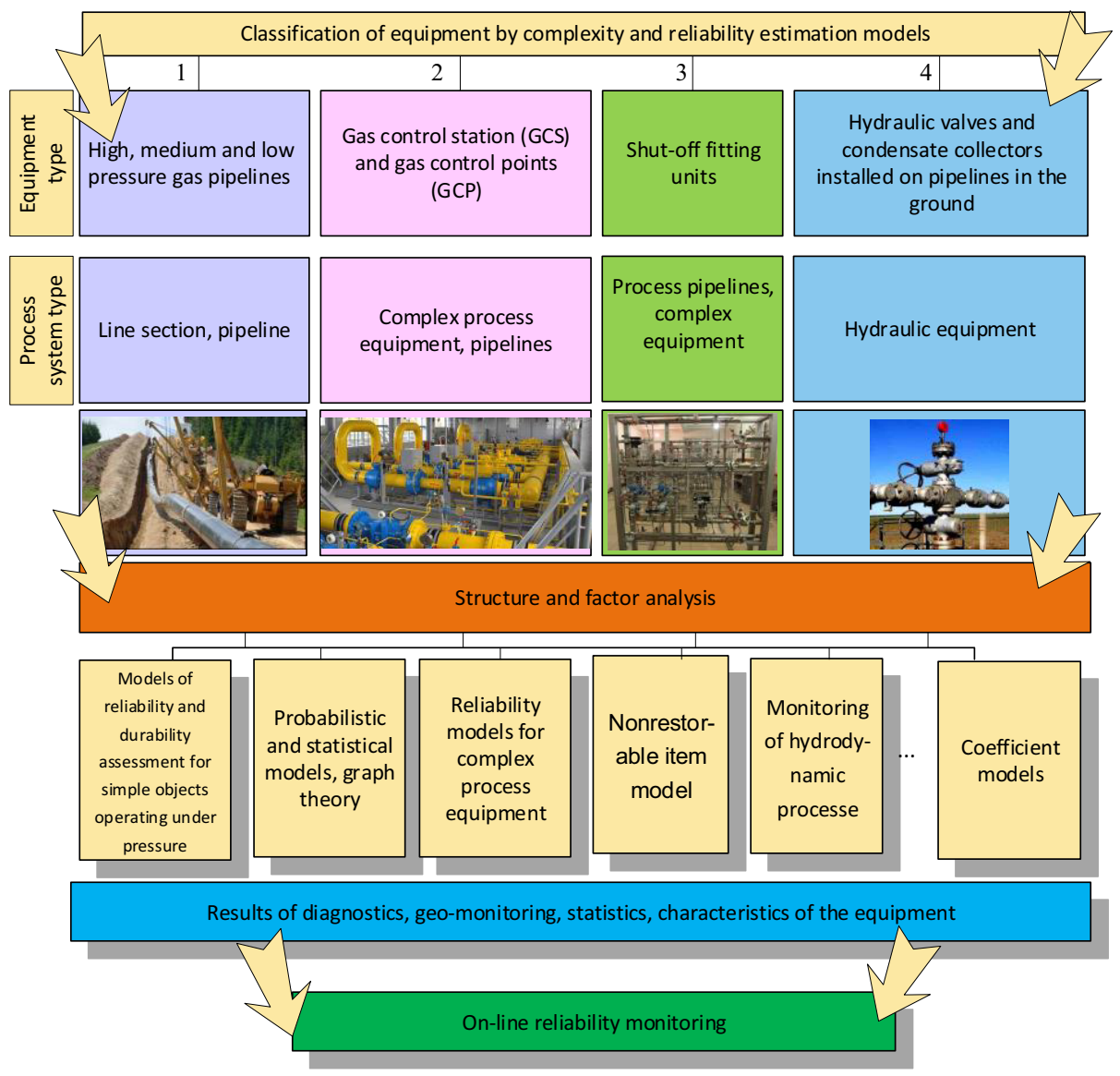

Fig.2.Classification scheme of technological equipment of gas distribution networks. 
When optimizing the maintenance system during reliability monitoring the process flow diagram and the number of GDP are of great importance. Since reconstruction of a gas distribution network is very costly, a special task is to determine the number of GDP and to choose the optimal strategy of gas distribution network maintenance.

The task of automatic redundancy and on-line maintenance is important. For example, the task of operative automatic redundancy can be posed and solved by using the following mathematical apparatus.

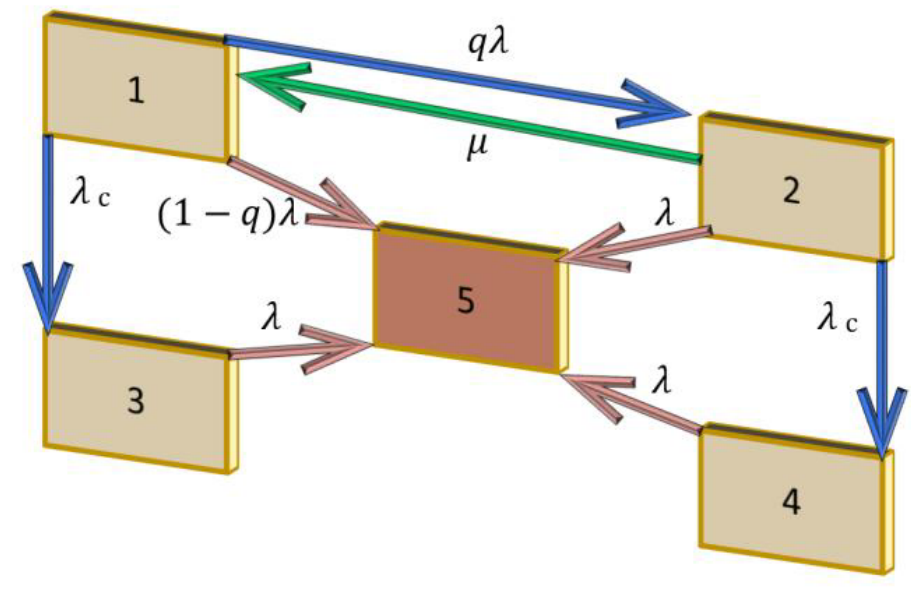

Fig.3. Graph of automatically duplicated system maintenance.

Duplication of technical systems is an effective way to ensure reliability. It is particularly efficient in the presence of automatic redundancy, which allows obtaining, under certain conditions, practically reliable systems even in the presence of automatic switching systems and timely detection of leaks. For example, maintenance of the system is illustrated by the graph in Figure 3. The system works smoothly if at least one of the subsystems is trouble-free. In case of a working subsystem refusal it is translated into recovery mode and a backup comes into operation. Subsystem recovery time is much shorter than time between failures. The reserve is considered unloaded. We take recovery as full. In the simplest mathematical model subsystems are equally reliable and are characterized by simple failure flows with intensity $\lambda$ and recoveries with intensity $\mu$.The intensity of control system failures $\lambda_{c}$, when assessing the effectiveness of the monitoring system, this figure can characterize the intensity of the prediction error. Fullness of subsystem control exercised by the indicator, $q \in(0,1)$.

Values of $\mathrm{q}$ are determined by the number of controlled system parameters from their complete plurality $\mathrm{n}$ :

$$
q=\frac{\lambda}{\lambda+\lambda_{\mathrm{c}}}
$$

At any given time with a probability $\operatorname{Pi}(\mathrm{t})$ the system can be in one of five states (Figure3). In the first state, all system nodes are trouble-free, in the second one subsystem failed, in the third a subsystem and switching indicator are faulty, in the fourth a switching indicator failed, in the fifth the system fails, this state is absorbing. Probability of fail-safe operation of such system $\operatorname{Pc}(\mathrm{t})$ and probability of it being in any of the first four states.

The average time of transition from the first state, including through the second, third, fourth into the absorbing will be time between system failures.

The system of differential equations for the maintenance graph looks like: 


$$
\left\{\begin{array}{c}
P_{1}^{\prime}(t)=-\left(\lambda_{\mathrm{c}}+\lambda\right) P_{1}(t)+\mu P_{2}(t), \\
P_{2}^{\prime}(t)=-\left(\mu+\lambda_{\mathrm{c}}+\lambda\right) P_{2}(t)+q \lambda P_{1}(t), \\
P_{3}^{\prime}(t)=-\lambda P_{3}(t)+\lambda_{\mathrm{c}} P_{2}(t), \\
P_{4}^{\prime}(t)=-\lambda P_{4}(t)+\lambda_{\mathrm{c}} P_{1}(t) .
\end{array}\right.
$$

As probability of fail-safe operation $P_{c}(t)=\sum_{i=1}^{n} P_{i}(t)$, then from (2) we find

$$
P_{\mathrm{c}}^{\prime}(t)=-\lambda P_{\mathrm{c}}(t)+q \lambda P_{1}(t) \text {. }
$$

The unknown factor $P_{1}(t)$ in (3) can be found from the first two Equations 2, containing $P_{1}(t)$ and $P_{2}(t)$. Suppose at $t=0$ the system is fail-safe, we $\operatorname{get} P_{l}(0)=1$, $P_{2}(0)=P_{3}(0)=P_{4}(0)=0$. Given the initial conditions, applying a direct Laplace transform $L_{i}(\alpha)=\int_{0}^{\infty} P_{i}(t) e^{\alpha t} d t$, we pass from these equations to algebraic:

$$
\left\{\begin{array}{c}
\left(\alpha+\lambda_{c}+\lambda\right) L_{1}(\alpha)-\mu L_{2}(\alpha)=1 \\
-q \lambda L_{1}(\alpha)+\left(\alpha+\mu+\lambda_{c}+\lambda\right) L_{2}(\alpha)=0
\end{array}\right.
$$

Now $P_{l}(t)$ can be written [1] as:

$$
P_{1}=\sum_{i=1}^{2} \frac{F_{1}\left(\alpha_{k}\right)}{F^{\prime}\left(\alpha_{k}\right)} e^{\alpha_{k} t}
$$

where

$$
F(\alpha)=\left|\begin{array}{cc}
\alpha+\lambda_{c}+\lambda & -\mu \\
-q \lambda & \alpha+\mu+\lambda_{c}+\lambda
\end{array}\right|,
$$

and $\alpha_{k}-$ the roots of $F(\alpha)=0$. The value $F_{1}\left(\alpha_{k}\right)$ is obtained in the case $\alpha=$ $\alpha_{k}$ from $F(\alpha)$ when replacing the first column with a column of the right sides of system (4). $F^{\prime}\left(\alpha_{k}\right)$ is a derivative of $F(\alpha)$ at $\alpha=\alpha_{k}$. Here $F_{1}\left(\alpha_{1}\right)=\alpha_{1}+\mu+\lambda_{\text {и }}+\lambda ; F_{1}\left(\alpha_{2}\right)=\alpha_{2}+$ $\mu+\lambda_{n}+\lambda ; F_{1}^{\prime}\left(\alpha_{1}\right)=\alpha_{1}-\alpha_{2} ; F_{1}^{\prime}\left(\alpha_{2}\right)=\alpha_{2}-\alpha_{1}$.

The roots of $F(\alpha)=0$ :

$$
\alpha_{1,2}=\left[-\left(\mu+2 \lambda_{\mathrm{c}}+2 \lambda\right) \pm \sqrt{\mu^{2}+4 q \mu \lambda}\right] / 2 .
$$

Thus, according to equation (5) we find

$$
\mathrm{P}_{1}(t)=A_{1} e^{\alpha_{1} t}+A_{2} e^{\alpha_{2} t},
$$

where

$$
\mathrm{A}_{1}=\frac{\alpha_{1}+\mu+\lambda_{\mathrm{c}}+\lambda}{\alpha_{1}-\alpha_{2}} ; \mathrm{A}_{2}=\frac{\alpha_{2}+\mu+\lambda_{\mathrm{c}}+\lambda}{\alpha_{2}-\alpha_{1}} / 2
$$

Solving equation (3) after substituting expression (7), we obtain the required probability of the fail-safe system operation, considering $P_{c}(0)=1$ :

$$
P_{c}(t)=q \lambda\left(\frac{A_{1}}{\alpha_{1}+\lambda} e^{\alpha_{1} t}+\frac{A_{2}}{\alpha_{2}+\lambda} e^{\alpha_{2} t}\right)+\left[1-q \lambda\left(\frac{A_{1}}{\alpha_{1}+\lambda}+\frac{A_{2}}{\alpha_{2}+\lambda}\right)\right] e^{-\lambda t} .
$$


To determine the meantime between failures $\mathrm{T}_{a v}^{-}$we transform the original data of equation (2) into a system of algebraic equations using the initial conditions and Laplace transform. Weget:

$$
\left\{\begin{array}{c}
\left(\alpha+\lambda_{c}+\lambda\right) L_{1}(\alpha)-\mu L_{2}(\alpha)=1, \\
-q \lambda L_{1}(\alpha)+\left(\alpha+\mu+\lambda_{\mathrm{c}}+\lambda\right) L_{2}(\alpha)=0, \\
-\lambda_{\mathrm{c}} L_{2}(\alpha)+(\alpha+\lambda) L_{3}(\alpha)=0, \\
-\lambda_{\mathrm{c}} L_{1}(\alpha)+(\alpha+\lambda) L_{4}(\alpha)=0 .
\end{array}\right.
$$

Summarizing equations (9), we find

$$
(\alpha+\lambda) L(\alpha)-q \lambda L_{1}(\alpha)=1
$$

Where $L(\alpha)=\sum_{i=1}^{4} L_{i}(\alpha)$.

Sincebydefinition

$$
\overline{\mathrm{T}}_{a v}=\int_{0}^{\infty} P_{c}(t) d t, \text { and } L(\alpha)=\int_{0}^{\infty} P(t) e^{-\alpha t} d t,
$$

Then $\overline{\mathrm{T}}_{\mathrm{c}}=L(0)$. But in case $\alpha=0$ Equation 10 looks like: $\quad \lambda L(0)-q \lambda L_{1}(\alpha 0)=1$,from which $\overline{\mathrm{T}}_{\mathrm{c}}=\frac{1}{\lambda}+q L_{1}(0)$. Value $L_{l}(0)$ can easily be determined from Equation 4 . Finally:

$$
\overline{\mathrm{T}}_{a v}=\frac{1}{\lambda}+q \frac{\mu+\lambda_{\mathrm{c}}+\lambda}{\left(\lambda_{\mathrm{c}}+\lambda\right)\left(\mu+\lambda_{\mathrm{c}}+\lambda\right)-q \lambda \mu} .
$$

Equation 11 is simplified taking into account the above mentioned real relations between the subsystem time between failures and its recovery time. As $\lambda$ and $\lambda_{c}$ are much less than $\mu$, the value

$$
\overline{\mathrm{T}}_{a v}=\frac{1}{\lambda}+\frac{q}{\left[\lambda_{\mathrm{c}}+(1-q) \lambda\right]} .
$$

In cases when the fullness of control $\mathrm{q}=1$

$$
\overline{\mathrm{T}}_{a v}=\overline{\mathrm{T}}_{s s}+\overline{\mathrm{T}}_{\mathrm{c}}
$$

The mean time to system failure is the sum of the mean time to failure of the subsystem $\mathrm{T}_{\mathrm{ss}}$ and cotrol system $\mathrm{T}_{\mathrm{c}}$.

The problem of optimizing the distribution of funds for maintenance and improvement of the reliability of gas network facilities is solved by dynamic programming, for example, using the well-known principle of optimality of Bellman with the objective function:

$$
F(x)=\max \left(\Delta N(x), C_{N}(x)\right)
$$




$$
\left\{\begin{array}{l}
F_{1,2}(A(t))=\max _{x \in d_{1}}\left[f_{1}(x, t)+f_{2}(A(t)-x)\right], \\
F_{1,2,3}(A(t))=\max _{x \in d_{2}}\left[F_{1,2}(x, t)+f_{3}(A(t)-x)\right], \\
\ldots \ldots \ldots \ldots \ldots \ldots \\
F_{1,2 \ldots . .(n-1)}(A(t))=\max _{x \in d_{n-1}}\left[F_{1,2 \ldots(n-2)}(x, t)+f_{n-1}(A(t)-x)\right], \\
F(A(t))=\max _{x \in d_{n}}\left[F_{1,2 \ldots(n-1)}(x, t)+f_{n}(A(t)-x)\right] .
\end{array}\right.
$$

Where $\mathrm{A}$ - the total funding; $\mathrm{x}_{1}, \mathrm{x}_{2} \ldots \mathrm{x}_{\mathrm{n}}$ - amounts of funding; $\mathrm{n}$ - the number of possible decisions on the distribution of funds; $F\left(x_{1}, x_{2} \ldots x_{n}\right)$ - the funds distribution function; $f_{1,2 \ldots n}$ - the reliability distribution function.

Functioning of the model in real time is possible in the case the following conditions are met:

$$
x_{1}+x_{2}+\ldots+x_{n}=A(t)
$$

Where functions $\mathrm{f}, \mathrm{A}-$ depend on time $\mathrm{t}$.

Solution of this system solves the problem of funds distribution granting the maximum system reliability.

\section{Results}

Figure 4 shows an example of the implementation of the technological equipment monitoring system of gas distribution networks. An important aspect of this system is the fact that at every system level - high, medium, low pressure - the operating organization has a possibility to control and plan the optimal service system taking into account the responsibility code. For example, when operating low pressure networks the responsibility code increases due to low-skilled consumers. For networks of medium and high pressure where automation tools and regular maintenance schedules are used and there is an operational recovery team, the level of responsibility is reduced.

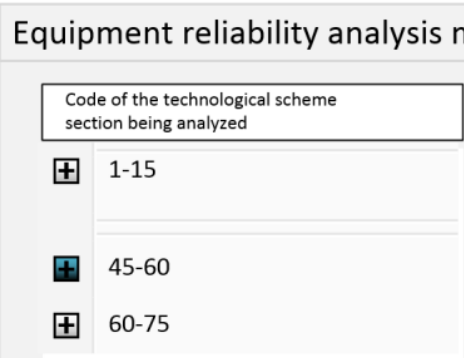

\section{M2 M3}

\begin{tabular}{|l|}
\hline Current state \\
\hline Recommended control point, hour \\
\hline 2100 \\
\hline Critical control point, hour \\
\hline 2400 \\
\hline
\end{tabular}

Fig.4. Example of the implementation of the technological equipment monitoring system of gas distribution networks.

When evaluating and planning indicators the use of factor analysis based on the graph theory $[4,5,11-14]$ is essential. It solves two main problems of monitoring: the need for a comprehensive and, at the same time, detailed consideration. Factor analysis enables identification of hidden variables responsible for linear statistical correlations between the observed variables. The graph theory allows us to solve the problem of optimizing gas transport systems. Analysis has shown that in some cases the quality of the initial 
information in the design, operation and reconstruction significantly affects the nature of selecting the reliability evaluation model.

\section{Discussion}

Analysis of existing sources indicates that, despite the urgency of the problem and a significant amount of research, the existing techniques allow for only one-time assessment of reliability indicators and are not intended to be used for the rapid assessment and forecasting in real time with the use of modern computer technology.

Models of reliability indicators and algorithms developed by the authors provide for the predictability of parameters of technical facilities in real time or over a fixed period of time, the function of the structure and factor system analysis in order to optimize maintenance and identify vulnerable elements.

\section{Conclusions}

A practical value of this work consists in the development of a complex of mathematical models and forecasting techniques for the decision support system, monitoring of reliability indicators, operating in real time and facilitating the transition to the system of preventive maintenance and repairs on the forecasted indicators of reliability.

\section{References}

1. A.N. Shipovalov, Y.D. Zemenkov, S.Y. Toropov, S.Y. Podorozhnikov, M.Y. Zemenkova, I.V. Tyrylgin, V.P. Pavlov, Aspects of technological reliability and costeffectiveness of operation of underground storage facilities of natural gas in Western Siberia(TSOGU, Tyumen,2012)

2. URL: http://expert.ru/2012/02/28/gaz-razrushitel/media/128077/

3. V.A. Petrjakov,M.Y. Zemenkova,M.A. Kulikov,A.N. Shipovalov,Modern problems of science and education 2(3), 34 (2015)

4. M.Y. Zemenkova, System monitoring of indicators of reliability of objects of pipeline transport(TSOGU, Tyumen, 2007)

5. M.Y. Zemenkova,A.N. Shipovalov,Y.D. Zemenkov,Materials Science and Engineering 127(1), 54 (2016) DOI: 10.1088/1757-899X/127/1/012056

6. Russian standard SP 62.13330.2011

7. A.A. Ionin, Gas supply (publ. h. "Lan", SPb,2012)

8. A.A. Bessonov, Forecasting of automatic system reliability performance (Energia,Leningrad, 1971)

9. M.G.Sukharev,Reliability of energy systems and equipment. Directory. Reliability of gas and oil supply systems (Nedra, Moscow,1994)

10. A.L. Pimnev, M.Y. Zemenkova, Y.D. Zemenkov, D.P. Iljyashchenko, Materials Science and Engineering 127(1), 68 (2016) DOI: 10.1088/1757-899X/127/1/012049

11. M. Zemenkova, V. Shalay, Y. Zemenkov, E. Kurushina, MATEC, 73 (2016) DOI: 10.1051/matecconf/20167307001

12. M.Yu.Zemenkova,Y.D. Zemenkov, A.L. Pimnev, E.V. Kurushina, Materials Science and Engineering 127(1), 54 (2016) DOI: 10.1088/1757-899X/127/1/012055 
13. V. Shalay, M. Zemenkova, Y. Zemenkov, S. Toropov,MATEC, 73(2016)DOI: $10.1051 /$ matecconf/20167301029

14. M. Zemenkova,Y. Zemenkov,A. Gladenko,S. Podorozhnikov,MATEC, 86 (2016)DOI: 10.1051/matecconf/20168604053 